\title{
Norepinephrine Inhibits Macrophage Migration by Decreasing CCR2 Expression
}

\author{
Fangming Xiu, Mile Stanojcic, Marc G. Jeschke*
}

Ross Tilley Burn Centre, Sunnybrook Health Science Centre, Sunnybrook Research Institute, Division of Plastic Surgery, Department of Surgery, Department of Immunology, University of Toronto, Toronto, Ontario, Canada

\begin{abstract}
Increased incidences of infectious and septic complications during post-burn courses represent the main contributor to burn injury mortality. Sustained increases in catecholamine levels, especially norepinephrine (NE), contribute to immune disturbances in severely burned patients. The precise mechanisms underlying NE-mediated immunoregulation are not fully understood. Here we hypothesize that persistently elevated NE levels are associated with immunodysfunctions. We examined the effects of NE on the phenotype and functions of bone marrow-derived macrophages (BMMs). Whole mouse bone marrow cells were treated in vitro with $40 \mathrm{ng} / \mathrm{mL}$ of $\mathrm{M}$-CSF and with $1 \mathrm{x}$ $10^{-6} \mathrm{M}$ or $1 \times 10^{-8} \mathrm{M}$ of NE or without NE for 7 days; cells were collected and stained with antibodies for CD11b, $\mathrm{F} 4 / 80, \mathrm{MHC}$ II and the inflammatory CC chemokine receptor 2 (CCR2). We found $1 \times 10^{-6} \mathrm{M}$ of NE inhibited MHC II and CCR2 expression on $\mathrm{CD} 11 \mathrm{~b}^{+} / \mathrm{F} 4 / 80^{+} \mathrm{BMM}$ cells. It also inhibited BMM proliferation by inhibiting CSF-1R expression. On the contrary, $1 \times 10^{-8} \mathrm{M}$ of NE slightly increased both MHC II and CCR2 expression on CD11b ${ }^{+}$ $\mathrm{F} 4 / 80^{+} \mathrm{BMM}$ cells but inhibited $\mathrm{CD} 11 \mathrm{~b}^{+} / \mathrm{F} 4 / 80^{+} \mathrm{BMM}$ proliferation. MCP-1 based migration assay showed that the migration of $1 \times 10^{-6} \mathrm{M}$ of NE-treated BMM toward MCP-1 was significantly decreased compared to BMM without NE treatment. Both $1 \times 10^{-8} \mathrm{M}$ and $1 \times 10^{-6} \mathrm{M}$ of NE enhanced TNF- $\alpha$ production and phagocytosis of FITC-Dextran. Intracellular staining of transcriptional factor MafB showed that $1 \times 10^{-6} \mathrm{M}$ of NE treatment enhanced its expression, whereas $1 \times 10^{-8} \mathrm{M}$ of NE decreased expression. Stimulation with LPS in the last 24-hours of BMM culture further decreased CCR2 and MHC II expression of these BMM, suggesting the synergistic effect of LPS and NE on macrophage. Our results demonstrate that NE regulates macrophage differentiation, proliferation and function, and may play a critical role in the dysfunctional immune response post-burn.
\end{abstract}

Citation: Xiu F, Stanojcic M, Jeschke MG (2013) Norepinephrine Inhibits Macrophage Migration by Decreasing CCR2 Expression. PLoS ONE 8(7): e69167. doi:10.1371/journal.pone.0069167

Editor: Pranela Rameshwar, University of Medicine and Dentistry of New Jersey, United States of America

Received May 21, 2013; Accepted June 11, 2013; Published July 2, 2013

Copyright: $\odot 2013$ Xiu et al. This is an open-access article distributed under the terms of the Creative Commons Attribution License, which permits unrestricted use, distribution, and reproduction in any medium, provided the original author and source are credited.

Funding: This work was supported by the following grant funding sources: Canadian Institutes of Health Research \# 123336; CFI Leader's Opportunity Fund: Project \# 25407. NIH RO1 GM087285-01; and Physicians' Services Incorporated Foundation - Health Research Grant Program. The funders had no role in study design, data collection and analysis, decision to publish or preparation of the manuscript.

Competing interests: The authors have declared that no competing interests exist.

${ }^{*}$ E-mail: marc.jeschke@sunnybrook.ca

\section{Introduction}

Despite major advances in the management of patient care following burn injury, the incidence of sepsis has significantly increased over the past two decades [1,2]. Burns covering more than $30 \%$ total burn surface area (TBSA) are associated with stress, inflammation, hypermetabolism and catabolism that lead to profound morbidity and mortality $[3,4]$. Burn injury is one of the most severe forms of trauma accompanied by stress responses. The stress response causes an immediate increase in catecholamines and in burn patients these levels can reach several folds, while persisting for prolonged periods [5,6]. Enhanced adrenergic stimulation and catecholamine release are important components of the pathophysiology of severe burn and sepsis. In an experimental model of burn sepsis, abrogation of bone marrow NE content with 6-OHDA resulted in a $62 \%$ survival rate compared to no survivors in NE-intact mice. Flow cytometric analysis of monocyte progenitors showed significantly more mature monocyte progenitors in NEdepleted mice [7], indicating that NE greatly influences monocyte maturation.

Phagocytes are an essential component of innate immunity and play an integral role in the immune response to burn injury. In severely burned and septic patients, myeloid commitment shifts toward monocytopoiesis [8,9] and dysfunctional macrophages $(M \Phi)$ are the hallmark of the disturbed immune response [10]. While a clear role norepinephrine plays in modulation of macrophages maturation $[7,11]$, there have been controversial results of catecholamine-mediated alterations in phagocytosis and TNF production. One study of murine wound showed that both low $\left(1 \times 10^{-9} \mathrm{M}\right)$ and high $\left(1 \times 10^{-6} \mathrm{M}\right)$ doses of NE suppressed wound macrophage phagocytic efficiency 
[12]. However, studies of mouse peritoneal macrophages found that lower doses of NE enhanced phagocytosis and cytokine production, whereas higher doses of NE have less effectiveness $[13,14]$. Macrophages are major producers of pro-inflammatory mediators during burn injury and sepsis [10]. Increased release of pro-inflammatory factors by macrophage appears to be of fundamental importance to the development of post-burn immune dysfunction, particularly at the early stage of disease [10]. On one hand, studies have shown that NE enhances TNF- $\alpha$ secretion from macrophage [15,16], whereas others show NE inhibiting TNF- $\alpha$ secretion from splenic macrophages isolated both from a polymicrobial sepsis mouse model and wild type rats $[17,18]$. These conflicting results further emphasize the demand for further investigation.

After burn injury, immune cells (ie. monocytes and neutrophils) infiltration into the wounded area is an important step of the healing process. In this process, chemokine receptor-dependent migration toward chemokine gradient is essential. A recent study found that CX3CR1 deficiency resulted in decreased recruitment of CX3CR1-positive myeloid cells into the burn wound leading to decreased wound healing [19]. Another study found that CCR2 was important for neutrophil tissue infiltration during sepsis [20]. This chemokine receptor pathway may be an attractive therapeutic approach for wound healing [21], however the specific role played by CCR2 on macrophage in severe burn and sepsis is yet to be examined.

Taking into consideration that the effects of NE on macrophage function are not fully understood, the present study investigates the effects of NE on macrophage proliferation, maturation and function in a murine bone marrow ex vivo culturing system. We found that NE has a broad regulating effect on macrophage differentiation, maturation and activities such as phagocytosis, migration and cytokine secretion. Our findings provide new insights into the mechanisms by which the catecholamines modulate the immune response in severely traumatized patients.

\section{Materials and Methods}

\section{Reagents and Abs}

FITC-Dextran (MW: 40K) and LPS from Escherichia coli 0111:B4 were purchased from Sigma-Aldrich (Oakville, Ontario, Canada). Recombinant murine M-CSF was purchased from PeproTech (Rocky Hill, NJ, USA) and stock solution of 40 $\mathrm{ng} / \mathrm{mL}$ was stored at $-80^{\circ} \mathrm{C}$. MCP-1 protein, RPMI 1640 lacking phenol red and charcoal-dextran-treated FBS were purchased from Invitrogen Life Technologies Inc. (Burlington, ON, Canada). BD Intracellular staining kit was from BD (Franklin Lakes, NJ, USA).

PE-conjugated rat anti-mouse CD11b, PerCPCy5.5conjugated rat anti-mouse F4/80 and APC-conjugated rat antimouse CSF-1 receptor were purchased from Biolegend (San Diego, CA, USA). FITC-conjugated mouse anti-mouse I-Ab was from BD (Franklin Lakes, NJ USA). APC-conjugated rat anti-mouse CCR2 was purchased from R\&D Systems (Minneapolis, MN, USA). FITC-conjugated TNF- $\alpha$ and rat $\lg \mathrm{G} 2 \mathrm{~b} \mathrm{k}$ isotope control were from eBioscience (San Diego,
CA, USA). MafB polyclonal primary antibody and FITCconjugated goat anti-rabbit IgG were from Abcam Inc. (Toronto, ON, Canada).

\section{Animal and bone marrow-derived macrophage culture}

All of the female C57BL/6 mice were purchased from Charles River Laboratories (St Constant, Quebec, Canada). All animals were housed maximum five per cage and maintained on a constant light: dark, 12:12 cycle. All animal procedures were approved by the Sunnybrook Health Science Center Animal Care Committee.

Murine bone marrow-derived macrophages were generated as previously described [22]. Bone marrow cells were prepared from femur and tibial bone marrow of C57BL/6 mice and the concentration was adjusted at $2 \times 10^{6} / \mathrm{mL}$. BMM cells were cultured in RPMI 1640 lacking phenol red supplemented with $40 \mathrm{ng} / \mathrm{mL}$ of mouse M-CSF, $2 \mathrm{mM}$ glutamine, $100 \mathrm{U}$ penicillin/ $0.1 \mathrm{mg}$ streptomycin $/ \mathrm{mL}, 10 \mathrm{mM}$ HEPES buffer, $10 \%$ of charcoal-dextran-treated FBS instead of regular FCS.

Total BM cells were seeded at $1 \times 10^{6}$ cells per $\mathrm{mL}$ in $2 \mathrm{~mL}$ of media in a 24-well plate and treated with or without NE. Various doses of NE (final concentrations, $1 \times 10^{-8} \mathrm{M}$ or $1 \times 10^{-6}$ $\mathrm{M})$ were added at day 0 . On day 3 and day 6 , half of the culture media were removed and replaced with fresh hormonedeficient media containing $40 \mathrm{ng} / \mathrm{mL}$ of M-CSF. Macrophages were harvested on day 7 . To activate macrophage, $50 \mathrm{ng} / \mathrm{mL}$ of LPS was added to the culture media at day 6 and cultured for another 24 hours. For the time point study, in addition to day 0 , NE (final concentration, $1 \times 10^{-6} \mathrm{M}$ ) was added on day 3 and day 6 . To evaluate the effects of NE on macrophage number, cells were counted immediately upon the completion of the cell culturing.

\section{Cell proliferation assay}

BMM proliferation regulated by NE was examined using the CyQUANT Cell Proliferation Assay Kit according to manufacturers instructions. Briefly, bone marrow cells were isolated as mentioned above and $2 \times 10^{5} \mathrm{BM}$ cells were seeded into each well of a 96-well plate and treated with various doses of $\mathrm{NE}$ (final concentrations, $1 \times 10^{-6} \mathrm{M}$ and $1 \times 10^{-8} \mathrm{M}$ ) or without NE. On day 7 , media were carefully removed, washed with PBS and plates were placed in a $-80^{\circ} \mathrm{C}$ freezer. The standard curve was made using BMMs harvested from culture flask in accordance with the protocol. Based on the standard curve, cell numbers in each sample can be calculated.

\section{Cell staining and flow cytometry}

After the seven day culture, cells were harvested after digestion with $10 \times$ Trypsin/EDTA at $37^{\circ} \mathrm{C}$ for $25 \mathrm{~min}$. To stain these cells, cells were incubated with Fc receptor blocker for 10 min at room temperature (RT) to block Fc receptors followed by phenotyping macrophage with antibodies for CD11b, F4/80, I$\mathrm{Ab}$, and CCR2 or CSF-1R in PBS containing 1\% BSA. Labeled cells were all run on the BD LSR II Flow Cytometer and data was analyzed using FlowJo (v. 8.7) software.

\section{Phagocytosis assay}

Phagocytosis of FITC-Dextran by macrophage was measured as the cellular uptake of FITC-dextran and quantified 
by Flow Cytometry. Bone marrow cells were isolated (as described in Generation of macrophage from BM cells) and treated with varying doses of NE (as described in Animal and bone marrow-derived macrophage culture). Treatment without any hormones served as a control. On day 7 of culturing, FITCDextran was added to the BMM culture at the final concentration of $200 \mu \mathrm{g} / \mathrm{mL}$ and incubated for $30 \mathrm{~min}$ at $37^{\circ} \mathrm{C}$. Cells added with the same amount of FITC-Dextran and incubated at $4^{\circ} \mathrm{C}$ for $30 \mathrm{~min}$ were used as the baseline of macrophage phagocytosis. All cells were collected and stained with PerCPCy5.5-conjugated anti-F4/80 antibody and PEconjugated anti-CD11b antibody. Internalization ability was evaluated at the percentage of FITC-positive cells gated on the $\mathrm{CD} 11 \mathrm{~b}^{+} \mathrm{F} 4 / 80^{+} \mathrm{BMMs}$.

\section{Intracellular staining of BMM}

Intracellular staining (ICS) of MafB was performed according to the direction of ICS kit (BD bioscience). Briefly, BMM cells were treated with NE (final concentrations, $1 \times 10^{-8} \mathrm{M}$ or $1 \mathrm{x}$ $10^{-6} \mathrm{M}$ ). On day 7 , the whole cells in each well were harvested. After Fc receptor blocking, cells were stained with antibodies for $F 4 / 80$ and $C D 11 b$. To do intracellular staining, cells were first resuspended with $100 \mu \mathrm{L}$ Fixation/Permeabilization solution for $30 \mathrm{~min}$ at $4^{\circ} \mathrm{C}$. Next, fixed and permeabilized cells were stained with the MafB primary antibody for $30 \mathrm{~min}$ at $4^{\circ} \mathrm{C}$. In the end, samples were stained with FITC-conjugated secondary antibody.

For intracellular cytokine staining (ICCS) of BMM, cells were cultured as before, LPS (final concentration, $50 \mathrm{ng} / \mathrm{mL}$ ) and BD GolgiPlug (final concentration, $1 \mu \mathrm{g} / \mathrm{mL}$ ) were added in the last 4 hours of culture. After Fc receptor blocking, cells were stained with antibodies for F4/80 and CD11b. To do intracellular TNF- $\alpha$ staining, permeabilized cells were stained with antibody for TNF- $\alpha$ or its corresponding isotype control for $30 \mathrm{~min}$ at $4^{\circ} \mathrm{C}$. TNF- $\alpha^{+}$BMMs were calculated by gating on CD11 ${ }^{+} \mathrm{F} 4 / 80^{+}$BMMs. Data were collected with a BD LSR II Flow Cytometer and analyzed by FlowJo software.

\section{Migration assay}

BM cells $\left(3 \times 10^{5}\right)$ were seeded on a 24-well Millicell $(8-\mu \mathrm{m}$ pore size filter) in $300 \mu \mathrm{L}$ media with $40 \mathrm{ng} / \mathrm{mL}$ M-CSF with NE (final concentrations, $1 \times 10^{-6} \mathrm{M}$ and $1 \times 10^{-8} \mathrm{M}$ ) or without NE treatment. The lower chamber was occupied with $500 \mu \mathrm{L}$ of the same media. At day 7 , the inserts were transfered into a new 24 well plate. The lower chamber was added with RPMI 1640 medium with $1 \%$ hormone-deficient serum and MCP-1 (the final concentration, $100 \mathrm{ng} / \mathrm{mL}$ ). Plates were incubated at $37^{\circ} \mathrm{C}$ for 4 hours. Cells in the lower chamber that passed through the filter were counted under a Carl Zeiss Primo Vert microscope (Carl Zeiss, Canada).

\section{Statistics}

All comparisons were performed using one-way ANOVA analysis using GraphPad Prism version 4 (GraphPad Software, San Diego CA), with a $p$ value less than 0.05 taken as statistically significant.

\section{Results}

\section{NE regulates macrophage differentiation and maturation from bone marrow}

We established a very stable ex vivo BMM culture system. After seven days culture with $40 \mathrm{ng} / \mathrm{mL}$ of M-CSF, over $95 \%$ of cells were $\mathrm{CD} 11 \mathrm{~b}^{+} / \mathrm{F} 4 / 80^{+}$(Figure S1B). To examine whether $\mathrm{NE}$ can regulate macrophage proliferation and maturation from bone marrow, we cultured bone marrow cells for 7 days with or without the treatment of NE. First, we determined BMM proliferation by CyQUANT Cell Proliferation Assay (Figure 1A). Our results showed that $1 \times 10^{-8} \mathrm{M}$ and $1 \times 10^{-6} \mathrm{M}$ of $\mathrm{NE}$ significantly inhibited macrophage proliferation $(p<0.05$ and $p<0.01$, respectively).

We next examined the phenotype of the cultured BMM on day 7. Cells were harvested and stained with antibodies for macrophage markers such as CD11b and F4/80, as well as maturation marker MHC II. At the end of the culture, most of the cells harvested were $\mathrm{CD} 11 \mathrm{~b}^{+} \mathrm{F} 4 / 80^{+} \mathrm{BMMs}$ without $\mathrm{NE}$ treatment. As represented in Figure $1 \mathrm{~B}$ and $1 \mathrm{C}$, based on the percentage of $\mathrm{MHC} \mathrm{II}^{+} / \mathrm{F} 4 / 80^{+} \mathrm{BMM}, 1 \times 10^{-6} \mathrm{M}$ of $\mathrm{NE}$ significantly inhibited $\mathrm{MHC}$ II expression compared to BMM without NE treatment $(13.2 \pm 2.7$ vs. $24.8 \pm 3.3 \%$, respectively, $\mathrm{p}<0.01)$. Interestingly, $1 \times 10^{-8} \mathrm{M}$ of NE slightly increased the expression of MHC II, but it was not statistically significant.

To determine whether the presence of NE was required during the entire 7-day culture period to promote BMM proliferation, $1 \times 10^{-6} \mathrm{M}$ of $\mathrm{NE}$ was added to $\mathrm{BM}$ cells on different days after isolation (day 0,3 , or 6 ) and remained in culture until the cells were harvested on day 7 . As shown in Figure $1 \mathrm{D}, 1 \times 10^{-6} \mathrm{M}$ of $\mathrm{NE}$ was most effective in inhibiting BMM maturation when added from day 0 of culture. The maturation-inhibiting effects of high doses of NE decreased daily as the culture progressed.

The inhibiting effects of NE on BMM proliferation may be attributed to decreased CSF-1R as shown in Figure 2. Compared to controls, both the percentage and MFI of CSF-1R ${ }^{+}$BMM treated with $1 \times 10^{-6} \mathrm{M}$ of NE were higher (Figure $2 \mathrm{~A}$ and $B)(p<0.01$ and $p<0.05$, respectively). We failed to detect an effect of low dose NE on CSF-1R expression (data not shown).

Taken together, our data showed the inhibiting effects of NE on BMM proliferation and maturation. High doses of NE (1 $\mathrm{x}$ $\left.10^{-6} \mathrm{M}\right)$ inhibited BMM proliferation and maturation, whereas low doses of NE $\left(1 \times 10^{-8} \mathrm{M}\right)$ only slightly increased $\mathrm{MHC}$ II expression. Based on the time course studied, it is likely that $\mathrm{NE}$ acts on every differentiation stage of BMMs.

\section{NE inhibits macrophage migration by decreasing CCR2 expression}

Chemokine receptors such as CCR2 are very important for monocyte/macrophage egressing from bone marrow to circulation and migrating into tissues during chronic inflammation or acute infection [23,24]. Thus, we determined the CCR2 expression of NE treated bone marrow-derived macrophages. As seen in Figure $3 \mathrm{~A}$ and $3 \mathrm{~B}, 1 \times 10^{-6} \mathrm{M}$ of NE significantly decreased CCR2 expression based on the percentage of $\mathrm{CCR}^{+} / \mathrm{F} 4 / 80^{+}$macrophages compared to 
A

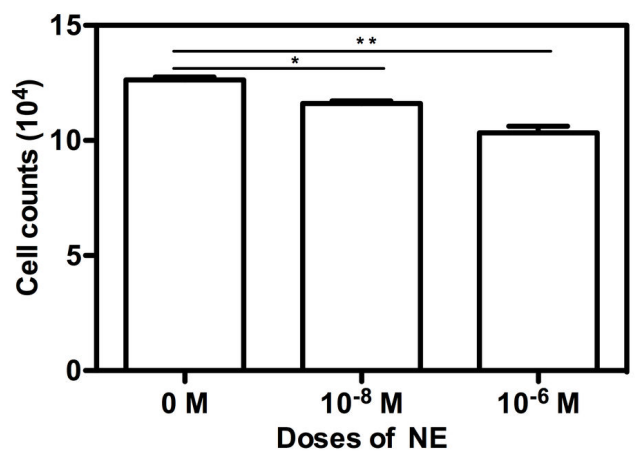

B

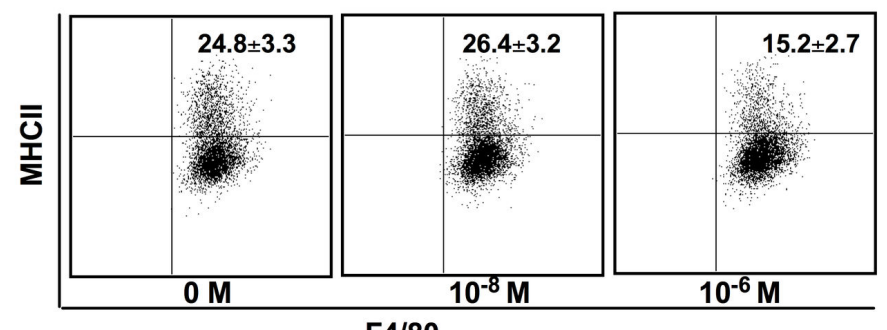

F4/80

C

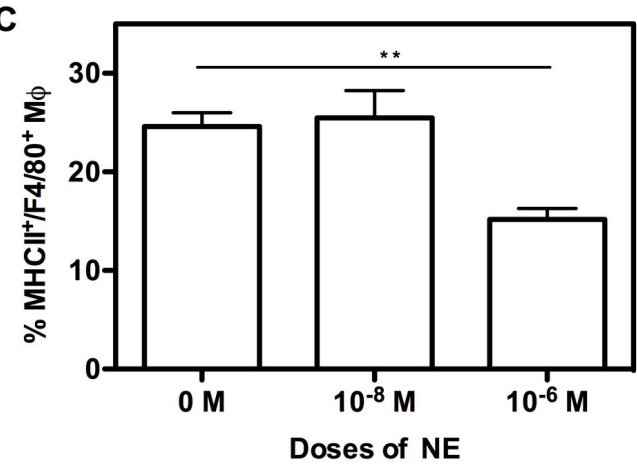

D

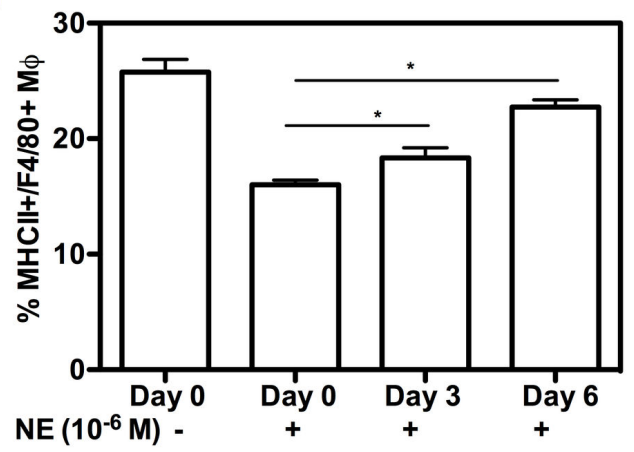

Figure 1. NE regulates macrophage proliferation and maturation from BM. Unfractionated BM cells were plated in a 24-well plate at $2 \times 10^{6}$ cells/well and cultured for 7 days in hormone-deficient medium with murine M-CSF alone, or in varying concentrations of NE $\left(1 \times 10^{-8} \mathrm{M}\right.$ or $\left.1 \times 10^{-6} \mathrm{M}\right)$ added at day 0 , or in the presence of $1 \times 10^{-6} \mathrm{M}$ of NE added at different time points (day 0, 3 and 6). BMM proliferation was examined using the CyQUANT Cell Proliferation Assay Kit. Briefly, $2 \times 10^{5}$ BM cells were seeded into each well of a 96-well plate and treated with various doses of NE or without NE. At day 7 , cells were collected and total cell numbers were counted based on the standard curve (A). To phenotype BMM, cells stained with Abs for CD11b, MHC II and $\mathrm{F} 4 / 80$. Representative dot plot data of the percentage of $\mathrm{MHC} \mathrm{I}+/ F 4 / 80^{+} \mathrm{M} \varphi$ are shown in (B). The graphic format of the percentage of $\mathrm{MHC} \mathrm{II} / \mathrm{F} 4 / 80^{+} \mathrm{M} \varphi$ is shown in (C). The time course of $\mathrm{NE}$ effects is shown in (D). Data show mean $\pm \mathrm{SD}$ of 4 independent experiments. Significant difference is indicated as ${ }^{*} p<0.05$ or ${ }^{* *} p<0.01$, compared to untreated control or different time points.

doi: 10.1371/journal.pone.0069167.g001 
A

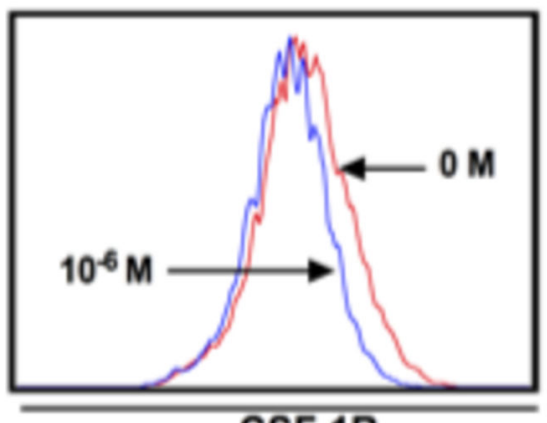

CSF-1R
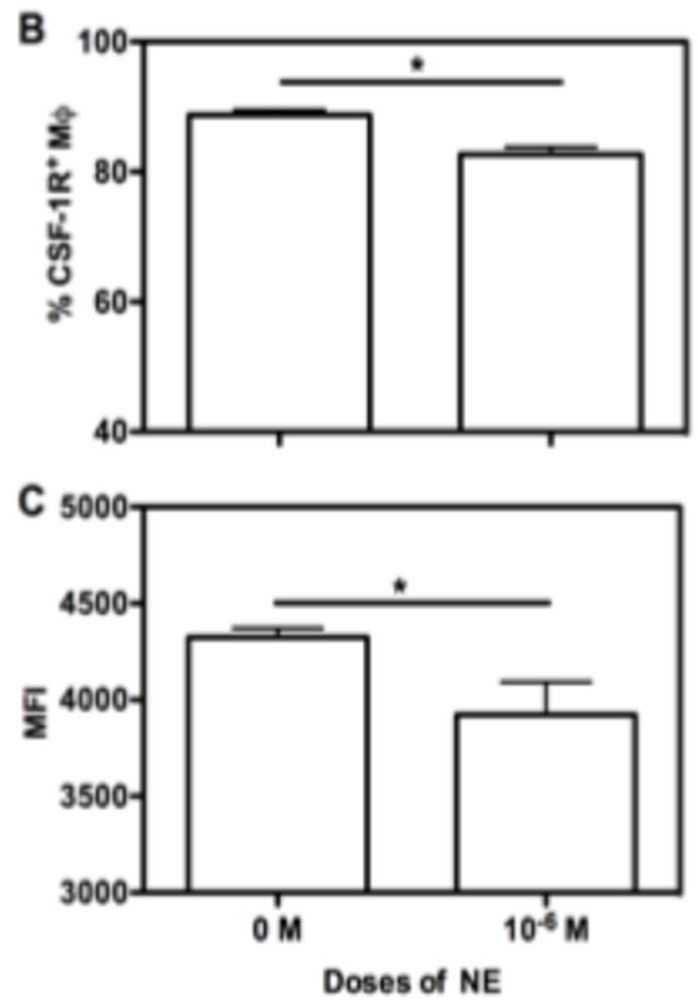

Figure 2. High dose of NE decreases CSF-1R expression. Unfractionated BM cells were cultured with murine M-CSF alone, or in the presence of varying concentrations of epinephrine. At day 7, cells were collected and stained with Abs for CD11b, F4/80 and CSF-1R. Based on the gating of $C D 11 b^{+} / F 4 / 80^{+}$BMM cells, representative histogram data of CSF-1R expression on $M \varphi$ is shown in (A). The corresponding graphic format data of CSF-1R expression on $M \varphi$ is shown in (B). Their corresponding MFI of CSF-1R expression on $M \varphi$ is shown in (C). Data show mean $\pm S D$ of 4 independent experiments. Significant difference is indicated as ${ }^{*} p<0.05$, compared to untreated control.

doi: 10.1371/journal.pone.0069167.g002

controls (39.2 \pm 4.7 vs. $24.1 \pm 2.9 \%, p<0.05)$. However, $1 \times 10^{-8}$ $\mathrm{M}$ of NE did not have significant effects on CCR2 expression.

To explore whether the decrease in CCR2 by $1 \times 10^{-6} \mathrm{M}$ of $\mathrm{NE}$ could lead to inhibited migration towards MCP-1, we

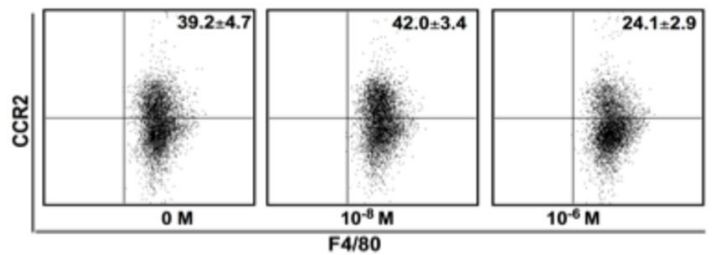

B
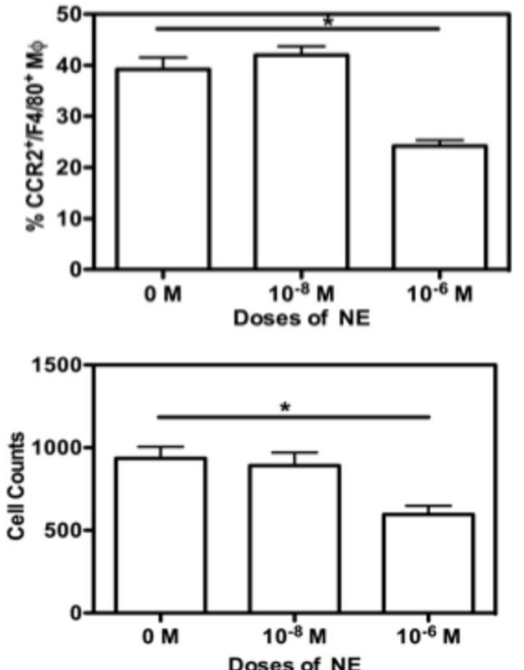

Figure 3. NE inhibits macrophage migration by decreasing CCR2 expression. BM cells were cultured in hormonedeficient medium with murine M-CSF alone, or in the presence of varying concentrations of NE. At day 7 , cells were collected and stained with Abs for CD11b, F4/80 and CCR2. Based on the gating of $C D 11 b^{+} / F 4 / 80^{+}$cells, representative dot plot for percentage of $\mathrm{CCR} 2^{+} / \mathrm{F} 4 / 80^{+} \mathrm{M} \varphi$ is shown in (A). The corresponding graphic format data of percentage of $\mathrm{CCR}^{+} /$ $\mathrm{F} 4 / 80^{+} \mathrm{M} \varphi$ is shown in (B). The comparison of cells that passed through insert filter after 4 hours incubation with MCP-1 (final concentration, $50 \mathrm{ng} / \mathrm{mL}$ ) in the lower chamber are shown in Figure 2 (C). Data show mean \pm SD of 4 independent experiments. Significant difference is indicated as ${ }^{*} p<0.05$, compared to untreated control.

doi: 10.1371/journal.pone.0069167.g003

utilizing the Transwell Migration Assay. After a 4 hour incubation, cells that migrated to the lower chamber were counted. As shown in Figure 3C, $1 \times 10^{-6} \mathrm{M}$ of NE treated BMM had significantly less cells compared to those without treatment or with $1 \times 10^{-8} \mathrm{M}$ of NE. Although $1 \times 10^{-8} \mathrm{M}$ of NE slightly increased CCR2 expression, it did not lead to altered migration. Therefore, only a high dose of NE $\left(1 \times 10^{-6} \mathrm{M}\right)$ has significant effects on CCR2 expression and migration of BMM.

\section{LPS exacerbates NE's effects}

It has been well documented that LPS down-regulates the CCR2 expression of macrophages [25,26]. Taking into consideration the known elevation in LPS in severely burned patients due to disrupted intestinal permeability [27], thus we explored whether LPS and high dose of NE $\left(1 \times 10^{-6} \mathrm{M}\right)$ have combinational effects. LPS $(50 \mathrm{ng} / \mathrm{mL})$ was added to the BMM 
culture $\left(1 \times 10^{-6} \mathrm{M}\right.$ or $\left.0 \mathrm{M}\right)$ at day 6 and cultured for 24 hours. We found that LPS had additional effects with high dose NE (1 $x 10^{-6} \mathrm{M}$ ) including $\mathrm{BMM}$ maturation and CCR2 expression. The

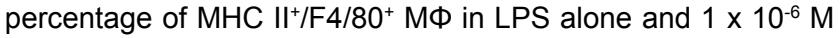
NE alone were $21.7 \pm 2.5$ and $16.9 \pm 2.3$, respectively, whereas it was $10.0 \pm 2.0$ in LPS with $1 \times 10^{-6} \mathrm{M}$ of NE (Figure $4 \mathrm{~A})$. The differences between the combination of $1 \times 10^{-6} \mathrm{M}$ of $\mathrm{NE}$ alone, LPS alone or both combined were statistically significant (Figure 4B) $(p<0.05$ and $p<0.01$, respectively). Similarly, compared to treatment with either LPS alone or NE alone, treatment with $1 \times 10^{-6} \mathrm{M}$ of NE plus LPS induced significantly lower expression of CCR2 in BMM (Figure 4C and D) $(30.1 \pm 3.0$ vs. $15.9 \pm 3.9,26.7 \pm 2.2$ vs. $15.9 \pm 3.9$, respectively). Taken together, our results demonstrated that LPS exacerbated NE's effect on the expression of MHC II and CCR2 on BMMs.

\section{NE promotes macrophage phagocytosis}

To examine whether BMM treated with NE had altered phagocytosis, FITC-Dextran was added to the BMM culture on day 7 for 30 minutes and FITC-Dextran ${ }^{+}$BMM were determined by FACs. As shown in Figure $5 A$ and $B$, both high and low doses of NE enhanced BMM phagocytosis of Dextran compared to treatment with $0 \mathrm{M}$ of $\mathrm{NE}$, in a dose-dependent manner. As the baseline control, the percentage of FITCDextran ${ }^{+} \mathrm{BMM}$ at $4^{\circ} \mathrm{C}$ was only $11 \%$ (data not shown). At $0 \mathrm{M}$, their were $42.8 \%$ Dextran $^{+}$BMM, whereas their were $58.9 \%$ and $69.6 \%$ for $1 \times 10^{-8} \mathrm{M}$ and $1 \times 10^{-6} \mathrm{M}$, respectively. The differences in the proportion of FITC-Dextran ${ }^{+}$BMM between 0 $\mathrm{M}$ and $1 \times 10^{-8} \mathrm{M}$ along with $1 \times 10^{-6} \mathrm{M}$ were statistically significant $(p<0.05$ and $p<0.01$, respectively). Our results demonstrated that both $1 \times 10^{-8} \mathrm{M}$ and $1 \times 10^{-6} \mathrm{M}$ NE enhanced the phagocytosis of BMMs in a dose-dependent manner.

\section{NE enhance TNF- $\alpha$ production}

Macrophages are a major source of many cytokines involved in immune response in burn and sepsis [10]. To examine whether NE had an effect on BMM cytokine secretion, we determined TNF- $\alpha$ secretion after LPS stimulation for 4 hours. As represented in Figure $6 \mathrm{~A}$ and $\mathrm{B}$, without NE treatment, there was only $45 \%$ of $C D 11 b^{+} / F 4 / 80^{+}$BMMs secreting TNF- $\alpha$. However, over $90 \%$ of NE-treated BMM secreted TNF- $\alpha$. The effects of NE peaked at $1 \times 10^{-8} \mathrm{M}$. A concentration of $1 \times 10^{-6}$ $\mathrm{M}$ did not further increase the percentage of TNF- $\alpha^{+}$BMM cells. Taken together, these findings suggest that adrenergic stimulation may influence peripheral tissue macrophage inflammatory cytokine response following trauma and sepsis.

\section{NE regulates MafB expression}

To investigate the mechanisms of NE's effects on BMM differentiation, proliferation and maturation, we determined the expression of MafB, one of the essential transcriptional factors for macrophage differentiation [28], in BMM cells. As shown in Figure $7 \mathrm{~A}$, without NE treatment, only $71.8 \pm 4.6 \%$ of $\mathrm{BMM}$ expressed MafB, whereas $1 \times 10^{-8} \mathrm{M}$ and $1 \times 10^{-6} \mathrm{M}$ groups were $55.3 \pm 3.3$ and $97.5 \pm 3.1 \%$, respectively. The difference between the control and the treatment with $1 \times 10^{-8} \mathrm{M}$ or $1 \mathrm{x}$ $10^{-6} M$ were statistically significant $(p<0.05$ and $p<0.01$,

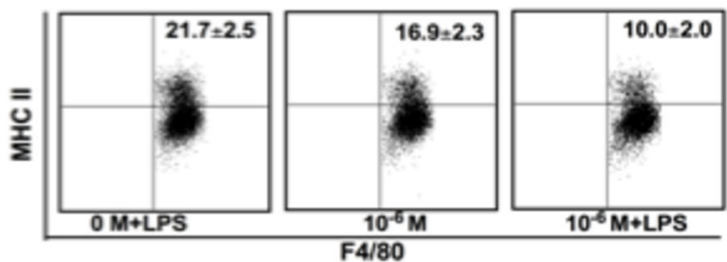

B

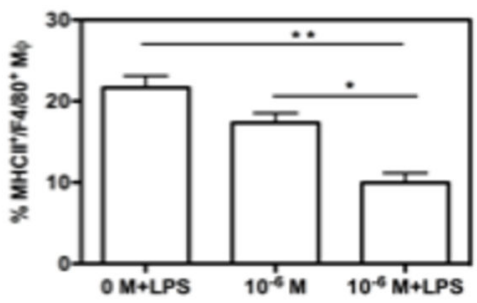

C

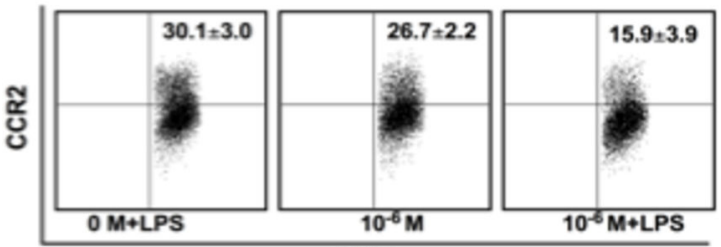

D

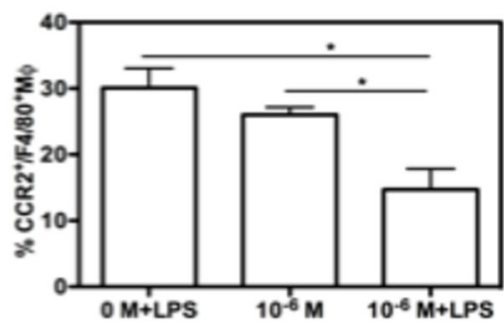

Figure 4. LPS exacerbates NE's effects. In the last 24 hours of 7 day culture of BM cells with the treatment of NE, LPS (50 $\mathrm{ng} / \mathrm{mL}$ ) was added to the culture. At the end of culture, cells were collected and stained with Abs for CD11b, MHC II and CCR2 antibodies. Representative dot plot data and their corresponding graphic format data of percentage of $\mathrm{MHC} \mathrm{I}^{+} /$ $\mathrm{F} 4 / 80^{+} \mathrm{M} \varphi$ are shown in (A) and (B) respectively. Representative dot plot data and their correspondent graphic format data of percentage of $\mathrm{CCR}^{+} / \mathrm{F} 4 / 80^{+} \mathrm{M} \varphi$ shown in (C) and (D) respectively. Data show mean \pm SD of 4 independent experiments. Significant difference is indicated as * $p<0.05$ and ${ }^{* *} p<0.01$, compared to untreated control. doi: 10.1371/journal.pone.0069167.g004

respectively). The MFI of $\mathrm{MafB}^{+} \mathrm{BMM}$ is the measure of MafB expression per cell. Compared to BMM without NE treatment, MFI of MafB ${ }^{+}$BMM treated with $1 \times 10^{-8} \mathrm{M}$ of NE or with $1 \times 10^{-6}$ of NE was significantly altered (Figure $7 C)(p<0.05$ and $p<0.01$, respectively). Since enhanced expression of MafB limits myeloid progenitor proliferation and accelerate macrophage differentiation [29], increased expression of MafB in $1 \times 10^{-6} \mathrm{M}$ of NE-treated BMM will push macrophage progenitors to mature quickly to macrophage, which leads to inhibited cell number as shown in Figure $1 \mathrm{~A}$. Overall, our results suggest 
A

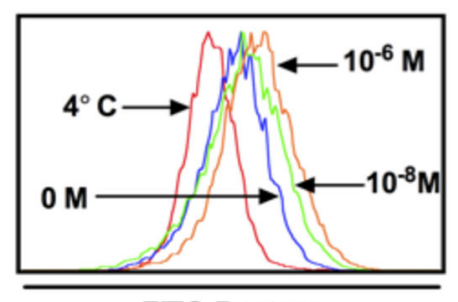

FITC-Dextran

B

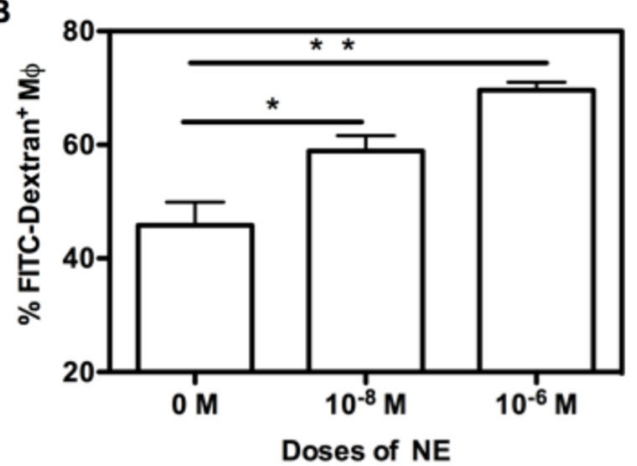

Figure 5. NE regulates antigen uptake by BMM. The whole bone marrow cells from C57BL/C mice were cultured with $M$ CSF in the presence of NE $\left(1 \times 10^{-8} \mathrm{M}\right.$ and $\left.1 \times 10^{-6} \mathrm{M}\right)$ for 7 days. At day 7 , FITC-Dextran $(200 \mu \mathrm{g} / \mathrm{mL})$ was added to the culture for further $30 \mathrm{~min}$ culture at $37^{\circ} \mathrm{C}$. As a negative control, FITC-Dextran-added wells were incubated at $4^{\circ} \mathrm{C}$ for $30 \mathrm{~min}$. At end of the experiment, cells were collected and stained with antibodies for CD11b and F4/80. The percentage of FITCDextran-positive CD11 $\mathrm{b}^{+} / \mathrm{F} 4 / 80^{+} \mathrm{BMM}$ was obtained by gating on $\mathrm{CD} 11 \mathrm{~b}^{+} / \mathrm{F} 4 / 80^{+} \mathrm{BMM}$. Representative flow cytometric data are shown in (A) and corresponding graphic data are shown in (B). Bars represent FITC-Dextran-positive DCs as the mean of 4 independent experiments $\pm S D$. ${ }^{*} p<0.05 ;{ }^{* *} p<0.01$, compared to untreated control $(0 \mathrm{M})$.

doi: 10.1371/journal.pone.0069167.g005

that NE may regulate macrophage differentiation, proliferation and maturation by regulating MafB expression.

\section{Discussion}

Dysfunctional macrophage is one of the hallmarks of severe burn and sepsis [10]. However, the mechanism underlying macrophage dysfunction during severe trauma and illness are not fully understood. In the present study, we explored the effects of NE on macrophage differentiation, maturation and function. Using the ex vivo bone marrow-derived macrophage culturing system, our study demonstrates that NE has comprehensive regulatory effects on macrophage differentiation, maturation and function. First, we showed that NE regulates BMM proliferation and maturation. Both high and low doses of NE inhibit BMM proliferation. However, only low dose of NE enhances BMM maturation as determined by $\mathrm{MHC}$ II and F4/80 expression. Secondly, high dose of NE $\left(1 \times 10^{-6} \mathrm{M}\right)$ regulates macrophage migration by decreasing CCR2
A

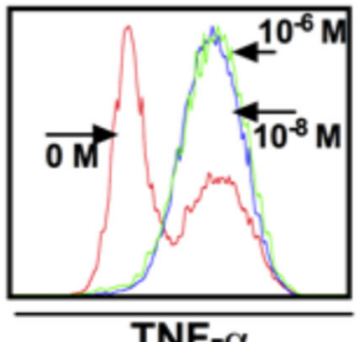

TNF- $\alpha$

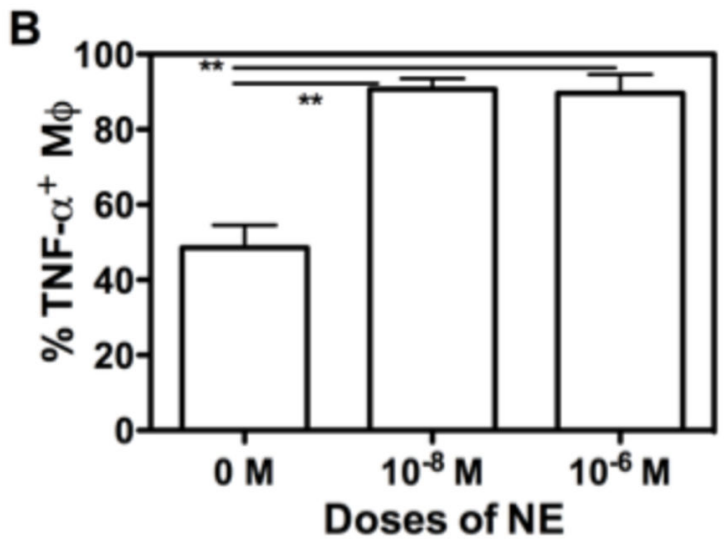

Figure 6. NE enhances TNF- $\alpha$ production from BMM. Bone marrow cells were cultured with M-CSF in the presence of NE $\left(1 \times 10^{-8} \mathrm{M}\right.$ and $\left.1 \times 10^{-6} \mathrm{M}\right)$. At day 7 , LPS $(50 \mathrm{ng} / \mathrm{mL})$ and the protein transport inhibitor GolgiPlug (final concentration, 1 $\mu \mathrm{g} / \mathrm{mL}$ ) were added to the culture for further 4 hour culture and then cells were collected and sequentially stained with membrane antibodies for CD11b and F4/80, and intracellular antibody of TNF- $\alpha$. Representative flow cytometric data was shown in (A) and corresponding graphic data in (B). Graph bars represent TNF- $\alpha^{+}$BMM as the means \pm SD of 2 independent experiments in triplicate each time. ${ }^{* *} p<0.01$, compared to untreated control (0 M).

doi: 10.1371/journal.pone.0069167.g006

expression. The migration of macrophages from circulation into tissue plays an essential role for wound healing. Our data suggest that NE may regulate tissue immune response by regulating CCR2-dependent monocyte tissue infiltration in severe burn and sepsis. We also showed that both $1 \times 10^{-8} \mathrm{M}$ and $1 \times 10^{-6} \mathrm{M}$ of NE enhanced macrophage phagocytosis. Since increased macrophage phagocytosis is essential for a faster wound healing, NE may promote wound healing in this regard. Fourth, both high and low doses of NE enhanced TNFa production. Macrophages are major producers of proinflammatory mediators following thermal injury and hyperactivity is of critical importance to the development of post-burn immune dysfunction, such as systemic inflammatory response syndrome (SIRS) [10]. Our results show that concentrations as low as $1 \times 10^{-8} \mathrm{M}$ NE significantly increase TNF- $\alpha$ production from BMM, under the stimulation of LPS. Furthermore, it indicates that catecholamines play a role in acute inflammation during burn. We also found that epinephrine has similar effects on macrophage but with a less 
A

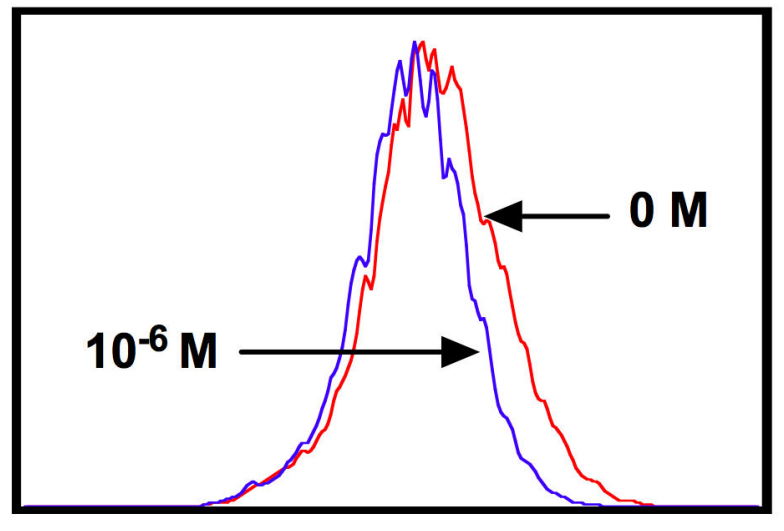

CSF-1R
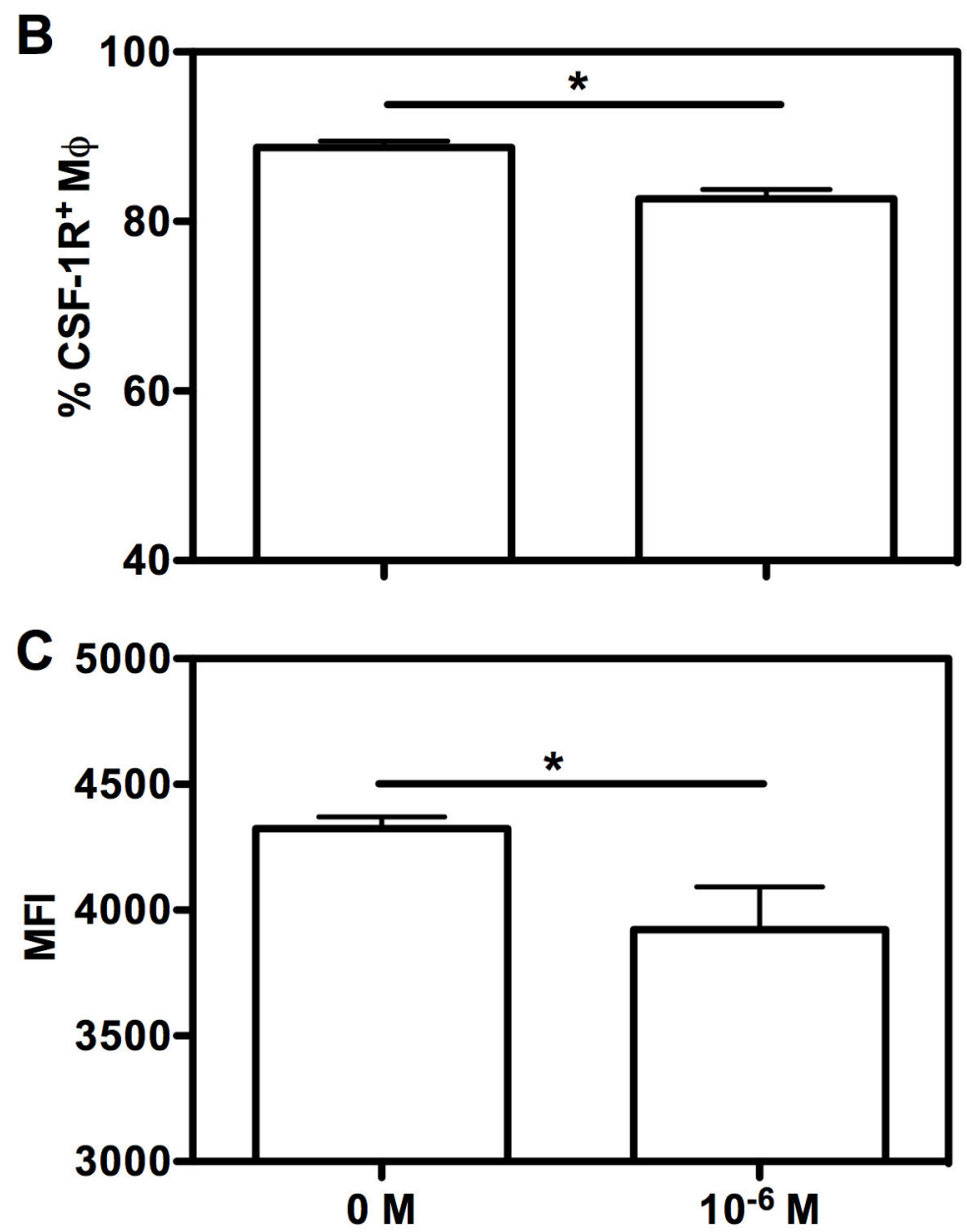

Doses of NE

Figure 7. NE regulates MafB expression of BMM. At the end of the 7 day BMM culture, cells were harvested and stained with antibodies for phenotypic markers CD11b and F4/80 followed by sequential intracellular staining with primary MafB antibody and FITC-conjugated secondary antibodies. Representative dot plot data of percentage of MafB ${ }^{+} \mathrm{BMM}$ were shown in (A) and their corresponding graphic format in (B). The MFI of $\mathrm{MafB}^{+} \mathrm{BMM}$ were shown in (C). Data represent samples in triplicate for each time of two independent determinations. ${ }^{*} p<0.05,{ }^{* *} p<0.01$, compared to non-hormone treated control $(0 \mathrm{M})$.

doi: 10.1371/journal.pone.0069167.g007 
efficient mode (Figure S2). In summary, our study suggests that catecholamines may play a significant role in disturbed immune response during severe burn and sepsis by regulating macrophage differentiation and function.

The modulatory effects of NE on macrophage function are particularly prominent in phagocytosis and cytokine secretion. For macrophage phagocytosis, our results are in contrast to previous studies [12,30]. One of those studies using macrophage isolated from burn wound showed that NE inhibited macrophage phagocytosis of Escherichia coli [12]. This discrepancy may be due to the activation status and source of macrophage used in the experiments. A study on the effects of stress on macrophage behaviours found that stress caused a decrease in phagocytosis mediated by Fc- $\gamma$ or mannose receptors in resting peritoneal macrophage, whereas increased phagocytosis in peritoneal macrophage isolated from mice that had been intraperitoneally injected with LPS 4 days before the experiment [31]. The increased cytokine secretion presently shown in our study is consistent with studies using thioglycollate-elicited mouse peritoneal macrophages [16], whereas other reports of NE modulation on cytokine function show decreases using normal rat spleen and CLP splenic macrophage $[17,18]$. Though we failed to show the dosedependent effect of NE on macrophage TNF- $\alpha$ production in our system, other researchers showed the difference between different doses. It was reported that low levels of NE result in enhanced production of TNF- $\alpha[15,16]$, whereas high levels of NE inhibit TNF- $\alpha$ production $[15,17]$. Therefore, the effect of NE on macrophage activity is largely dependent on the activation status and source of macrophage as well the doses of NE.

LPS or endotoxin is an important structural component of the outer membrane of Gram-negative bacteria and is one of the best studied among pathogen-associated molecular pattern of bacteria. Nearly three decades ago, Maejima et al. showed that serious injury led to translocation of enteric bacteria into the mesenteric lymph nodes and liver because of gut barrier disruption [27]. Dr. Mannick's laboratory further extended the notion that circulating LPS triggered the systemic inflammatory response syndrome in severe trauma and burn injury [32]. LPS has been shown to decrease CCR2 expression of macrophage $[25,33]$, however the combinatory effects of LPS and catecholamines on macrophage function have not yet been studied. Our results demonstrated that LPS exacerbates NE's effects on macrophage maturation and CCR2 expression. Therefore, in severe trauma and sepsis, when both LPS and catecholamine are increased, collective exposure will worsen the microenvironment for monocyte progenitors differentiation and maturation. In addition, macrophage activity can be sensitized by endotoxin. Assessing the effect of burn insult on endotoxin shock, Enomoto et al. found that endotoxin released from permeabilized intestine exacerbates burn outcome by sensitizing Kupffer cells [34,35]. Our own results showed that in the presence of LPS, NE's effect peaks at $1 \times 10^{-8} \mathrm{M}$, Further increases in NE dose did not enhance the production of TNF- $\alpha$. Taken together, this suggests that the interactions between LPS and catecholamine are essential to regulating macrophage activities during severe trauma.

Macrophage is an important member of the mononuclear phagocyte system (MPS), which includes monocyte, macrophage and DC. Cells in the bone marrow that are committed to MPS are the macrophage and DC progenitor (MDP). Lineage commitment and differentiation of bone marrow progenitors toward MPS is tightly orchestrated by specific transcription factors and essential cytokines [36] and this is dysregulated in sepsis [37-40]. The pool of Lin- Sca-1+ c-kit+ (LSK) cells, which are enriched in bone marrow hematopoietic stem cells, is expanded in a sepsis model [37]. Although it has not been known whether bone marrow hematopoietic stem cells (HSC) sense infection directly, it does respond to LPS [39]. Both LPS signaling and reduction in bone marrow cellularity alone can induce the expansion of bone marrow hematopoietic stem and progenitor cells [37,38]. Our results suggest that catecholamine may act directly on macrophage progenitors since $1 \times 10^{-6} \mathrm{M}$ of NE was most effective in inhibiting BMM maturation when added from day 0 of culture (Figure 1D). Due to the higher expression of transcription factors like MafB and receptor for M-CSF, GMPs is skewed to favor monocyte commitment [40] [8]. In contrast to their results, our data showed that higher expression of MafB (Figure 7) and decreased expression of CSF-1R (Figure 2) on high dose of NE-treated BMMs contribute to inhibited differentiation and proliferation of BMMs. The emergency myelopoietic response to severe trauma redirects progenitor differentiation in severely burned and septic patients, but the differentiation toward macrophage and DC fails in time for chronically inflamed patients.

In conclusion, our findings on the effects of catecholamines on macrophage differentiation and function are important. This study was the first to show that catecholamines regulate CCR2 expression in BMMs. Our results not only provide greater insight towards understanding the pathophysiology of severe burn and sepsis, but also raise some concerns regarding immunotherapies targeting CCR2 in septic patients. A recent report found that CCR2 is essential for neutrophil infiltration during sepsis and suggested that targeting CCR2 might be a novel immunotherapy for sepsis [21]. However, our results present some inherited challenges. Due to the dual role of NE on CCR2 expression in macrophages, caution should be taken when targeting CCR2 in sepsis. Future studies should extend the current findings and examine CCR2 expression on monocytes/macrophages in an animal model or clinical patients.

\section{Supporting Information}

\section{Figure S1. Gating schemes.}

Unfractionated BM cells were plated in a 24-well plate at $2 \mathrm{x}$ $10^{6}$ cells/well and cultured for 7 days in hormone-deficient medium with murine M-CSF alone. At day 7 , cells were collected and stained with Abs for CD11b and F4/80. Representative SSC/FSC is shown in (A) and the percentage of $\mathrm{CD} 11 \mathrm{~b}^{+} / \mathrm{F} 4 / 80^{+} \mathrm{M} \varphi$ in the culture without NE treatment is shown in $(B)$.

(TIF)

Figure S2. Epinephrine regulates MHC II and CCR2 expression of BMM. 
Unfractionated BM cells were plated in a 24-well plate at $2 \mathrm{x}$ $10^{6}$ cells/well and cultured for 7 days in hormone-deficient medium with murine $\mathrm{M}-\mathrm{CSF}$ alone, or in varying concentrations of epinephrine $\left(1 \times 10^{-7} \mathrm{M}\right.$ or $\left.1 \times 10^{-5} \mathrm{M}\right)$ added at day 0 . At day 7, cells were collected and stained with Abs for CD11b, MHC II, CCR2 and F4/80. Representative dot plot data of the percentage of $\mathrm{MHC} \mathrm{II}^{+} / \mathrm{F} 4 / 80^{+} \mathrm{M} \varphi$ and $\mathrm{CCR} 2^{+} / \mathrm{F} 4 / 80^{+} \mathrm{M} \varphi$ are shown in $(A)$ and $(C)$, respectively. The graphic format data of the percentage of $M H C \mathrm{II}^{+} / \mathrm{F} 4 / 80^{+} \mathrm{M} \varphi$ and $\mathrm{CCR}^{+} / \mathrm{F} 4 / 80^{+} \mathrm{M} \varphi$ are shown in (B) and (D), respectively. Data show mean \pm SD of 4 independent experiments. Significant difference is indicated as * $p<0.05$, compared to untreated control.

\section{References}

1. Koo DJ, Chaudry IH, Wang P (2000) Mechanism of hepatocellular dysfunction during sepsis: the role of gut-derived norepinephrine (review). Int J Mol Med,5: 457-465. PubMed: 10762647

2. Church D, Elsayed S, Reid O, Winston B, Lindsay R (2006) Burn wound infections. Clin Microbiol Rev,19: 403-434. doi:10.1128/CMR. 19.2.403-434.2006. PubMed: 16614255.

3. Kraft R, Herndon DN, Al-Mousawi AM, Williams FN, Finnerty CC et al. (2012) Burn size and survival probability in paediatric patients in modern burn care: a prospective observational cohort study. Lancet, 379: 1013-1021. doi:10.1016/S0140-6736(11)61345-7. PubMed: 22296810.

4. Jeschke MG, Mlcak RP, Finnerty CC, Norbury WB, Gauglitz GG et al. (2007) Burn size determines the inflammatory and hypermetabolic response. Crit Care,11: R90. doi:10.1186/cc5877. PubMed: 17716366.

5. Kulp GA, Herndon DN, Lee JO, Suman OE, Jeschke MG (2010) Extent and magnitude of catecholamine surge in pediatric burned patients. Shock,33: 369-374. doi:10.1097/SHK.0b013e3181b92340. PubMed: 20407405.

6. Jeschke MG, Gauglitz GG, Kulp GA, Finnerty CC, Williams FN et al. (2011) Long-term persistance of the pathophysiologic response to severe burn injury. PLOS ONE,6: e21245. doi:10.1371/journal.pone. 0021245. PubMed: 21789167

7. Tang Y, Shankar R, Gamboa M, Desai S, Gamelli RL et al. (2001) Norepinephrine modulates myelopoiesis after experimental thermal injury with sepsis. Ann Surg,233: 266-275. doi: 10.1097/00000658-200102000-00017. PubMed: 11176134.

8. Howell K, Posluszny J, He LK, Szilagyi A, Halerz J et al. (2012) High MafB expression following burn augments monocyte commitment and inhibits DC differentiation in hemopoietic progenitors. J Leukoc Biol,91: 69-81. doi:10.1189/jlb.0711338. PubMed: 21984745.

9. Santangelo S, Gamelli RL, Shankar R (2001) Myeloid commitment shifts toward monocytopoiesis after thermal injury and sepsis. Ann Surg,233: 97-106. doi:10.1097/00000658-200101000-00015. PubMed: 11141231.

10. Schwacha MG (2003) Macrophages and post-burn immune dysfunction. Burns,29: 1-14. doi:10.1016/S0305-4179(02)00187-0. PubMed: 12543039.

11. Cohen MJ, Shankar R, Stevenson J, Fernandez R, Gamelli RL et al. (2004) Bone marrow norepinephrine mediates development of functionally different macrophages after thermal injury and sepsis. Ann Surg,240: 132-141. doi:10.1097/01.sla.0000130724.84914.d6. PubMed: 15213629.

12. Gosain A, Muthu K, Gamelli RL, DiPietro LA (2007) Norepinephrine suppresses wound macrophage phagocytic efficiency through alphaand beta-adrenoreceptor dependent pathways. Surgery,142: 170-179. doi:10.1016/j.surg.2007.04.015. PubMed: 17689682.

13. Muthu K, He LK, Szilagyi A, Strotmon P, Gamelli RL et al. (2010) ssadrenergic stimulation increases macrophage CD14 expression and E. coli phagocytosis through PKA signaling mechanisms. J Leukoc Biol, 88: 715-724. doi:10.1189/jlb.0410186. PubMed: 20643814.

14. Baccan GC, Sesti-Costa R, Chedraoui-Silva S, Mantovani B (2010) Effects of cold stress, corticosterone and catecholamines on phagocytosis in mice: differences between resting and activated macrophages. Neuroimmunomodulation,17: 379-385. doi: 10.1159/000292058. PubMed: 20516719.

15. Szelényi J, Kiss JP, Vizi ES (2000) Differential involvement of sympathetic nervous system and immune system in the modulation of TNF-alpha production by alpha2- and beta-adrenoceptors in mice. J Neuroimmunol,103: 34-40. doi:10.1016/S0165-5728(99)00234-9. PubMed: 10674987
(TIF)

\section{Author Contributions}

Conceived and designed the experiments: MJ FX MS. Performed the experiments: MJ FX MS. Analyzed the data: FX. Contributed reagents/materials/analysis tools: FX. Wrote the manuscript: FX MJ . Other: Designed the study: FX. Did all the experiments: FX.

16. Spengler RN, Allen RM, Remick DG, Strieter RM, Kunkel SL (1990) Stimulation of alpha-adrenergic receptor augments the production of macrophage-derived tumor necrosis factor. J Immunol,145: 1430-1434. PubMed: 2166759.

17. Hu XX, Goldmuntz EA, Brosnan CF (1991) The effect of norepinephrine on endotoxin-mediated macrophage activation. J Neuroimmunol,31: 35-42. doi:10.1016/0165-5728(91)90084-K. PubMed: 1845768.

18. Deng J, Muthu K, Gamelli R, Shankar R, Jones SB (2004) Adrenergic modulation of splenic macrophage cytokine release in polymicrobial sepsis. Am J Physiol Cell Physiol,287: C730-C736. doi:10.1152/ajpcell. 00562.2003. PubMed: 15151906.

19. Clover AJ, Kumar AH, Caplice NM (2011) Deficiency of CX3CR1 delays burn wound healing and is associated with reduced myeloid cell recruitment and decreased sub-dermal angiogenesis. Burns,37: 1386-1393. doi:10.1016/j.burns.2011.08.001. PubMed: 21924836.

20. Souto FO, Alves-Filho JC, Turato WM, Auxiliadora-Martins M, BasileFilho A et al. (2011) Essential role of CCR2 in neutrophil tissue infiltration and multiple organ dysfunction in sepsis. Am J Respir Crit Care Med,183: 234-242. doi:10.1164/rccm.201003-0416OC. PubMed: 20732989.

21. Kapoor A, Thiemermann C (2011) Targeting CCR2: a novel therapeutic strategy for septic shock? Am J Respir Crit Care Med,183: 150-151. doi:10.1164/rccm.201009-1403ED. PubMed: 21242593.

22. Weischenfeldt J, Porse B (2008) Bone Marrow-Derived Macrophages (BMM): Isolation and Applications. CSH Protoc, 2008: pdb prot5080

23. Engel DR, Maurer J, Tittel AP, Weisheit C, Cavlar T et al. (2008) CCR2 mediates homeostatic and inflammatory release of $\mathrm{Gr} 1$ (high) monocytes from the bone marrow, but is dispensable for bladder infiltration in bacterial urinary tract infection. J Immunol,181: 5579-5586. PubMed: 18832716

24. Tsou CL, Peters W, Si Y, Slaymaker S, Aslanian AM et al. (2007) Critical roles for CCR2 and MCP-3 in monocyte mobilization from bone marrow and recruitment to inflammatory sites. J Clin Invest,117: 902-909. doi:10.1172/JCI29919. PubMed: 17364026.

25. Sica A, Saccani A, Borsatti A, Power CA, Wells TN et al. (1997) Bacterial lipopolysaccharide rapidly inhibits expression of C-C chemokine receptors in human monocytes. J Exp Med,185: 969-974. doi:10.1084/jem.185.5.969. PubMed: 9120403.

26. Yi L, Chandrasekaran P, Venkatesan S (2012) TLR signaling paralyzes monocyte chemotaxis through synergized effects of p38 MAPK and global Rap-1 activation. PLOS ONE,7: e30404. doi:10.1371/ journal.pone.0030404. PubMed: 22347375.

27. Maejima K, Deitch EA, Berg RD (1984) Bacterial translocation from the gastrointestinal tracts of rats receiving thermal injury. Infect Immun,43: 6-10. PubMed: 6360909.

28. Kelly LM, Englmeier U, Lafon I, Sieweke MH, Graf T (2000) MafB is an inducer of monocytic differentiation. EMBO J,19: 1987-1997. doi: 10.1093/emboj/19.9.1987. PubMed: 10790365.

29. Sarrazin S, Mossadegh-Keller N, Fukao T, Aziz A, Mourcin F et al. (2009) MafB restricts M-CSF-dependent myeloid commitment divisions of hematopoietic stem cells. Cell,138: 300-313. doi:10.1016/j.cell. 2009.04.057. PubMed: 19632180.

30. Gosain A, Gamelli RL, DiPietro LA (2009) Norepinephrine-mediated suppression of phagocytosis by wound neutrophils. J Surg Res,152: 311-318. doi:10.1016/j.jss.2008.05.001. PubMed: 18952237.

31. Sesti-Costa R, Baccan GC, Chedraoui-Silva S, Mantovani B (2010) Effects of acute cold stress on phagocytosis of apoptotic cells: the role of corticosterone. Neuroimmunomodulation, 17: 79-87. doi: 10.1159/000258690. PubMed: 19923852 
32. Kelly JL, O'Sullivan C, O'Riordain M, O'Riordain D, Lyons A et al. (1997) Is circulating endotoxin the trigger for the systemic inflammatory response syndrome seen after injury? Ann Surg,225: 530-541. doi: 10.1097/00000658-199705000-00010. PubMed: 9193181.

33. Zhou Y, Yang Y, Warr G, Bravo R (1999) LPS down-regulates the expression of chemokine receptor CCR2 in mice and abolishes macrophage infiltration in acute inflammation. J Leukoc Biol,65: 265-269. PubMed: 10088610.

34. Williams JG, Bankey P, Minei JP, Mclntyre K, Turbeville T (1994) Burn injury enhances alveolar macrophage endotoxin sensitivity. J Burn Care Rehabil,15: 493-498. doi:10.1097/00004630-199411000-00006. PubMed: 7852452

35. Enomoto N, Takei Y, Yamashina S, Fukuda T, Suzuki S et al. (2004) Burn injury sensitizes rat Kupffer cells via mechanisms dependent on gut-derived endotoxin. J Gastroenterol,39: 1175-1181. doi:10.1007/ s00535-004-1468-9. PubMed: 15622482

36. Geissmann F, Manz MG, Jung S, Sieweke MH, Merad M et al. (2010) Development of monocytes, macrophages, and dendritic cells. Science, 327: 656-661. doi:10.1126/science.1178331. PubMed: 20133564.
37. Rodriguez S, Chora A, Goumnerov B, Mumaw C, Goebel WS et al. (2009) Dysfunctional expansion of hematopoietic stem cells and block of myeloid differentiation in lethal sepsis. Blood,114: 4064-4076. doi: 10.1182/blood-2009-04-214916. PubMed: 19696201.

38. Scumpia PO, Kelly-Scumpia KM, Delano MJ, Weinstein JS, Cuenca AG et al. (2010) Cutting edge: bacterial infection induces hematopoietic stem and progenitor cell expansion in the absence of TLR signaling. $J$ Immunol,184: 2247-2251. doi:10.4049/jimmunol.0903652. PubMed: 20130216.

39. King KY, Goodell MA (2011) Inflammatory modulation of HSCs: viewing the HSC as a foundation for the immune response. Nat Rev Immunol, 11: 685-692. doi:10.1038/nri3062. PubMed: 21904387.

40. Muthu K, He LK, Melstrom K, Szilagyi A, Gamelli RL et al. (2008) Perturbed bone marrow monocyte development following burn injury and sepsis promote hyporesponsive monocytes. J Burn Care Res,29: 12-21. PubMed: 18182893. 